\title{
STUDIES ON THE DISPOSITION FOR THE CARRIER OF BACILLUS THIAMINOLYTICUS MATSUKAWA ET MISAWA.
}

\author{
III. THE EFFECT OF BILE AND BILE ACIDS FROM VARIOUS \\ ANIMALS ON THE INFECTION OF BACILLUS \\ THIAMINOLYTICUS MATSUKAWA ET MISAWA
}

KOYATA HAMADA

Research Laboratoly, Biofermin Co., Inc., Nagata-ku, Kobe

(Received December 24, 1955)

It was reported in the previous report (1), that the sheep and rabbit, both being herbivora, are the most resistant animals to BMM, while the cat, a carnivore, is apt to become a carrier of the bacillus. This seems to agree with the finding of Horikawa (2) that thiaminase disease patients generally like meat diets. The experiments made by the author (1) as well as by Horikawa (2), however, indicate that a meat diet has no effect on the production of thiaminase disease, and that the diet acts merely as an external factor on the disposition of BMM carriers. It was thus presumed that the difference in the disposition for the bacillus of various species of animals may be due to an internal factor.

Assuming that bile and bile acids might be associated with the interna1 factor, experiments were conducted to test their action on BMM. It has been reported that the animals, whose bile acids consist mainly of desoxycholic acid, can hardly become carriers of Salmonella, while those with bile acids consisting mainly of cholic acid easily become carriers, though transitorily (3). It has also been pointed out that the bile and bile acids exert physiologically a disinfectant effect on some bacteria in the intestinal tract, while they promote the growth of some other bacteria (4).

The present experiment has led to an noteworthy finding concerning the relationship between the disposition for BMM carriers and the kinds of bile acids.

\section{EXPERIMENTAL AND RESULTS}

\section{Inhibitory Activity of the Biles from Various Animals on the Growth} of $B M M$.

The rabbit, sheep, fowl, guinea-pig and cat were used as test animals. Their feces were preliminarily confirmed to be free from BMM by culture. 
The biles collected from five to ten animals were mixed and tested for the inhibitory activity on the growth of BMM, using a cup method.

The rabbit bile showed, as was

TABLE I

Inhibition by the Biles of Various Animals.

\begin{tabular}{l|c}
\hline \multicolumn{1}{c|}{ Animal } & $\begin{array}{c}\text { Average size of an } \\
\text { inhibitory zone }\end{array}$ \\
\hline Rabbit & $m \boldsymbol{m}$ \\
Sheep & 25.5 \\
Hen & 18.4 \\
Guinea - pig & 14.3 \\
Cat & 13.2 \\
& 10.2 \\
\hline
\end{tabular}
listed in Table I, the most strong inhibitory activity, followed, in a decreasing order, by the sheep, hen, guinea-pig and cat. This order is roughly the reverse of the liability to thiaminase disease reported in the preceding paper (1). Thus, the constituents of bile are assumed to be closely related with the disposition for $\mathrm{BMM}$ carriers.

\section{Inhibitory Activity of Bile Acids from Various Animals on the Growth} of $B M M$.

The inhibitory activity of the bile acids from the above-mentioned animals as well as several other bile acids were tested by a cup method.

All of the samples were used as sodium salts and homosulfanilamide (5), known as one of the strongest disinfectants against BMM, was also used for comparative study.

Desoxycholic Acid - It is contained in the bile of the rabbit and sheep. The acid used in this experiment was isolated from sheep's bile and purified by the method of Wieland et al. (6).

Chenodesoxycholic Acid - It is the chief bile acid in the bile of the hen and guinea-pig. The material used was isolated and purified by Shimizu's method (7).

Ursodesoxycholic Acid ${ }^{1}$ - It is the major bile acid of the bear.

Acetic Acid-Choleic Acid ${ }^{1}$ - This is a complex compound of desoxycholic and acetic acids, not occurring in nature.

Dehydrocholic Acid - It is formed in vivo by the oxidation of cholic acid. A preparation of Tanabe Pharmaceutical Co. Ltd., in ampoules, was used.

Cholic Acid - This is the chief constitute of the bile acids of the cat and sheep. The material used was isolated from the bile of the cat and purified by the method of Wieland $\boldsymbol{e t}$ al. (6).

Glycocholic and Taurocholic Acids - A commercial product of Minophagen Pharmaceutical Co. Ltd. was used.

The experimental results are summarized in Table II. Desoxycholic acid has showed a strikingly powerful inhibitory effect on BMM, more than that of homosulfanilamide, followed by chenodesoxycholic acid and ursodesoxycholic acid. Considering that the size of an inhibitory zone is usually proportional to the logarithm of the concentration of the effective substance in the cup method, the inhibitory activities of these substances may be con-

1 Kindly supplied by Dr. Tayei Shimizu, Okayama University Medical School. 
sidered to be moderate. Cholic, glycocholic and taurocholic acids were found to be least effective.

It may be concluded that the inhibitory activities of bile acids from various animals are closely associated with the tendency to become carriers of BMM.

TABLE II

Inhibitory Activity of the Bile Acids from Various Animals on the Growth of BMM. The figures indicate the average sizes of inhibitory zones in $\mathrm{mm}$.

\begin{tabular}{|c|c|c|c|c|c|c|c|c|c|c|c|c|c|c|}
\hline Bile acid & 10 & $10 \times 2$ & $10 \times 2^{2}$ & $10 \times 2^{3}$ & $10 \times 2^{4}$ & $10 \times 2^{5}$ & $10 \times 2^{a}$ & $10 \times 2^{7}$ & $10 \times 2^{8}$ & $10 \times 2^{9}$ & $10 \times 210$ & $10 \times 2: 1$ & $10 \times 212$ & $10 \times 2^{13}$ \\
\hline Desoxycholic acid & 33.0 & 32.0 & 31.0 & 30.5 & 30 & 27 & 23 & 20.5 & 14.5 & 10.5 & 9 & 9 & 8 & - \\
\hline Chenodesoxycholic acid & & 30.5 & 28.0 & 26.5 & 25.5 & 24.5 & 20 & 18 & 18 & 12 & 8 & 8 & - & - \\
\hline Ursodesoxycholic acid & 29.7 & 26.0 & 25.5 & 24.5 & 22 & 20 & 17 & 13 & 11 & 9 & 8 & 8 & - & 一 \\
\hline Acetic acid-choleic acid & & 26.5 & 24.5 & 23.5 & 21.5 & 18.5 & 17 & 13.5 & & & & & & \\
\hline Dehydrocholic acid & 26.5 & $\approx 2$ & 17.5 & 13 & 9 & 8 & - & - & - & & & & & \\
\hline Cholic acid & 20.2 & 16.7 & 12.7 & 11.2 & 9.7 & 8 & 8 & - & - & $\rightarrow$ & & & & \\
\hline Glycocholic acid & 18.5 & 15 & 14 & 11 & 10 & 9 & 8 & - & - & - & & & & \\
\hline Taurocholic acid & 12.0 & 11.7 & 11.0 & 9.2 & 9.0 & 8 & 8 & - & - & $\rightarrow$ & & & & \\
\hline Homosulfanilamide & 31 & 29 & 26 & 23.5 & 20 & 15 & 9.5 & 8 & 8 & - & - & - & - & - \\
\hline
\end{tabular}

\section{DISCUSSION}

The inhibitory effect of bilesand bile acids from various animals on the growth of BMM has been shown in the above experiments to differ considerably according to species. These results are compatible with the liabilities of animals to become carriers of BMM.

In man the bile consists chiefly of cholic, desoxycholic and chenodesoxycholic acids, and the composing ratio of the three acids may vary from one individual to another so that this may cause variations in the disposition for BMM. The amount of secretion of bile may play another role in it.

Though very many studies have been made concerning the attitude of bile and bile acids to bacteria, no bacterium is known to be so sensitive to various bile acids as BMM. That is, desoxycholic and chenodesoxycholic acids are more intensive in inhibitory activity than homosulfanilamide whereas the activity of cholic acid is remarkable in a high concentration, but falls promptly with dilution. Yamasaki (3) made a study on the disposition for the carriers of Salmonella group, and observed that the bile of the carriers was generally more diluted but richer in mucin as compared with the bile of healthy subjects. The author's observation that the intestinal microbes of the subjects disposed to become carriers of BMM are quite different in species from those of healthy subjects regardless of the presence of BMM, may possibly be due to the difference in the composition of bile and bile acids. 
1. Desoxycholic acid was shown to have an especially strong inhibitory action on the growth of BMM, chenodesoxycholic and ursodesoxycholic acids a moderate action, and cholic, glycocholic and taurocholic acids a very weak action.

2. This fact may explain why the disposition to become BMM carriers varies in different species of animals.

3. The individual difference in man in the disposition to become carriers may possively be explained by the difference in the amount of bile and the relative ratio of individual bile acids.

\section{ACKNOWLEDGEMENTS}

The author wishes to express his heartfelt thanks to Professor Hideo Katagiri, Kyoto University and Professor Masaji Tomita, Kobe University for encouragement, to Professor Akiji Fujita, Kyoto Prefectural University for encouragement and revision of the paper, to Professor Tayei Shimizu, Okayama University and Dr. Danji Matsukawa, Niigata University Medical School for valuable advices, and to Miss Fukiko Aoki and Mr. Masami Sasaki of the author's laboratory for an energetic assistance in experiments.

\section{REFERENCES}

1. Hamada, K., J. Vitaminol. 2, 72 (1956).

2. Horikawa, Y., Vitamins 4, 119 (1951).

3. Yamasaki, S., Seikagaku 2, 119 (1950).

4. Kitamura, N., J. Okayama Med. Ass. 65, 743 (1953).

5. Matsukawa, D., and Misawa, H., Proc. Vitamin B Res Comm. 35, 8 (1949).

6. Wieland. H., and Seibert, W., Z. Physiol. Chem. 262, 1 (1939).

7. Shimizu, T., in Kagakujikkengaku II, Vol. 10, p. 493, Kawade, Tokyo, 1939. 